\title{
Planar optical tweezers using tapered-waveguide junctions
}

\author{
Hong Cai and Andrew W. Poon* \\ Photonic Device Laboratory, Department of Electronic and Computer Engineering The Hong Kong University \\ of Science and Technology, Clear Water Bay, Hong Kong SAR, China \\ *Corresponding author: eeawpoon@ust.hk
}

Received May 4, 2012; revised June 8, 2012; accepted June 8, 2012; posted June 8, 2012 (Doc. ID 167973); published July 13, 2012

\begin{abstract}
We demonstrate planar optical tweezers using the evanescent field of a silicon nitride tapered-waveguide junction between a singlemode waveguide and a multimode waveguide. Our experiments show that the junction embedded in a fluidic channel holds up to one and two polystyrene particles of sizes of $2.2 \mu \mathrm{m}$ and $1 \mu \mathrm{m}$, respectively. The trapped particles are successively substituted by the incoming particles. Our experiments and numerical modeling reveal that the junction particle trapping depends on particle size and number. (c) 2012 Optical Society of America OCIS codes: $230.3120,230.7370,170.4520$.
\end{abstract}

Utilizing the surface wave of planar integrated photonic devices to optically manipulate micro/nano-sized objects has been gaining increasing interest in the research field of optofluidics for lab-on-a-chip applications. Several research groups demonstrated optical manipulation and sorting of particles using integrated waveguides [1,2]. Microresonators and nanoresonators have also been employed for more functional particle manipulation devices [3-8]. However, the reported particle trapping on planar devices requires standing wave from resonators $[\underline{7}, \underline{8}]$.

Here, we report planar optical tweezers with trapping and successive substitution of waveguided dielectric microparticles in static fluid on a silicon nitride $(\mathrm{SiN})$ tapered-waveguide junction connecting a singlemode waveguide taper to a multimode waveguide. The optical trapping mechanism is based on force balancing between the forward scattering force and the backward gradient force [9-12] induced by the surface wave distribution around the junction. Our experimental results and numerical modeling reveal that the junction particle trapping depends on the particle size and number.

Figures $1(\mathrm{a})-1(\mathrm{k})$ schematically show the principle of trapping and successive substitution of particles near a tapered-waveguide junction (with a tapering angle of $\sim 2^{\circ}$ ). Figure 1 (a) shows that particles are waveguided in the $z$ direction by the evanescent field on the junction top surface. In the vicinity of the junction $(z=0)$, the mode-field distribution diverges from a tightly confined mode in the singlemode waveguide to a less confined mode in the multimode waveguide [Fig. 1(b)]. The mode-field intensity of the surface wave gradually reduces in the taper region and drops on the multimode waveguide side $(z>0)$ [Fig. 1(c)].

In the Rayleigh particle model [9], we expect the particle in the vicinity of the junction to experience a gradient force $\left(F_{s}(z) \propto r^{3} \nabla I\right)$ in the $-z$ direction and a reducing scattering force $\left(F_{s}(z) \propto r^{6} I\right)$ in the $+z$ direction, where $r$ is the particle radius and $I$ is the evanescent field intensity. The total optical force upon a particle is given as $F_{\mathrm{oz}}=F_{g}+F_{s}$ [Fig. 1(d)]. $F_{\mathrm{oz}}$ near the junction can result in local equilibrium upon balancing $F_{s}$ and $F_{g}$ at $F_{\mathrm{oz}}(z)=0$, giving rise to a particle trap. By integrating the $F_{\mathrm{oz}}$ along the displacement from the first $F_{\mathrm{oz}}=0$, we obtain the potential energy $E(z)$ showing a potential well with a potential barrier [Fig. 1(e)]. The potential well $(E=0)$ is located near the junction at the first $F_{\mathrm{oz}}=0$. The potential barrier is located at the second $F_{\mathrm{oz}}=0$, with the barrier height given by the area of the $F_{\text {oz }}<0$ curve.

With an increasing particle size, the particle experiences a larger enhancement in $F_{s}\left(\propto r^{6}\right)$ than in $F_{g}\left(\propto r^{3}\right)$. Thus, the $F_{\text {oz }}$ becomes overall more positive $\left[F_{\text {oz } 2}\right.$ in Fig. 1(f)] and the corresponding potential energy well width and barrier height reduce [Fig. 1(g)]. In the case that the particle is sufficiently large that the $F_{\text {oz }}$-curve is shifted to above zero $\left[F_{\mathrm{oz} 3}\right.$ in Fig. 1(f)], the junction can no longer trap the particle [Fig. $\underline{1(\mathrm{~h})}$ ].
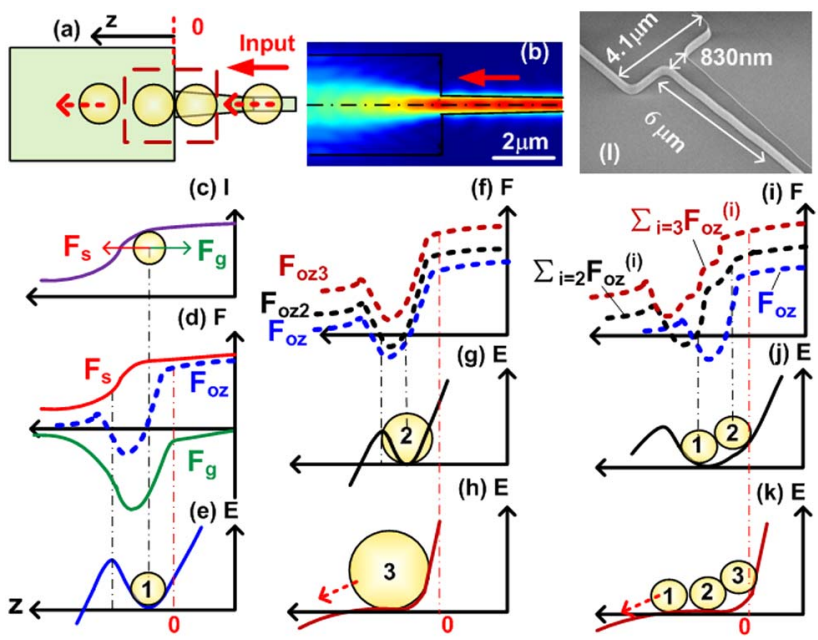

Fig.1. (Color online) (a) Schematic of particle trapping and successive substitution on a tapered-waveguide junction. Dashed-line window, junction region; (b) simulated mode-field amplitude distribution of the junction; (c)-(e) schematics of (c) the mode-field intensity profile along the dashed line in (b), (d) the optical forces, and (e) the potential energy of single Rayleigh particle 1; (f)-(h) schematics of the optical forces and the potential energy for single particles of increasing sizes. Size $1<2<3 . F_{\mathrm{oz} 2}: F_{\mathrm{oz}}$ on particle size $2 . F_{\mathrm{oz} 3}: F_{\mathrm{oz}}$ on particle size 3. Dashed arrow denotes particle escaping. (i)-(k) Schematics of the optical forces and the potential energy for multiple particles of a fixed size. $\sum_{i=2} F_{\mathrm{oz}}^{(i)}$, total $F_{\mathrm{oz}}$ on two particles. $\sum_{i=3} F_{\mathrm{oz}}^{(i)}$, total $F_{\mathrm{oz}}$ on three particles. (1) SEM picture of the fabricated device. 
With multiple particles of fixed size trapped in contact with each other, we expect the total force on the particles becomes a vector sum of the constitutive $F_{\text {oz }}$ on each particle, namely $\sum F_{\text {oz }}$ [Fig. 1(i)]. Here, we assume the scattering light from the particles is not significantly changing the waveguide field distribution. The total length of the trapped particle train is limited by the optical field divergence and the geometric limitation around the junction, similar to the focal depth in the conventional three-dimensional optical tweezers [11]. Thus, with an increasing number of particles to fill out the junction area, the total $\sum F_{\text {oz }}$ shifts upward [Fig. 1(i)].

Figure 1(j) shows a two-particle system with reduced potential barrier comparing to single particle [Fig. 1(e)]. When a new particle (\#3) arrives at the trapping site, the total $\sum F_{\mathrm{oz}}$ further shifts above zero, and the resulting potential barrier diminishes [Fig. 1(k)]. The junction cannot hold the three particles and the front-most particle (\#1) driven by its individual optical force first escape to the multimode region. The remaining particles (\#2 and \#3) form a new two-particle system being trapped upon the potential barrier restored.

In order to demonstrate such planar optical tweezers, we fabricate $0.7 \mu \mathrm{m}$ thick SiN tapered-waveguide junctions on a silicon wafer following our previous work $[\underline{3}, \underline{6}]$. Figure 1(1) shows the scanning electron microscope (SEM) picture of our junction. The input-waveguide is $\sim 0.4 \mu \mathrm{m}$ wide coupled to the multimode waveguide of $\sim 4.1 \mu \mathrm{m}$ wide by a taper of $6 \mu \mathrm{m}$ long (taper output end is $\sim 830 \mathrm{~nm}$ wide).

We end-fire $\sim 95-400 \mathrm{~mW}$ TM-polarized (E-field $\perp$ chip) laser light at $1550 \mathrm{~nm}$ wavelength into the tapered inputwaveguide using a polarization-maintaining lensed fiber. Our measurement shows an insertion loss of $\sim 15 \mathrm{~dB}$ with the upper-cladding of $1 \mu \mathrm{m}$ sized polystyrene particles (Polysciences) diluted in deionized water $\left(\sim 16.2 \times 10^{7}\right.$ particles $\left./ \mathrm{ml}\right)$. We estimate the coupling loss to be $\sim 7 \mathrm{~dB}$ [6] . The estimated guided power (EGP) in the input-waveguide is $\sim 7.5-32 \mathrm{~mW}$ near the junction, which is located at $\sim 3 \mathrm{~mm}$ from the input-waveguide end-face.

For $1 \mu \mathrm{m}$ sized particles, the junction traps one or two particles according to the guided power. Figures 2(a)-2(d) show the optical micrographs of the junction with $1 \mu \mathrm{m}$ sized particles at various times upon the EGP of $\sim 12 \mathrm{~mW}$. The junction traps only one particle (\#1) around $z=0$ [Fig. 2(a)]. As another particle (\#2) arrives [Fig. 2(b)], particle \#2 substitutes particle \#1, which moves forward to the multimode waveguide [Fig. 2(c)]. Particle \#2 is then trapped instead [Fig. 2(d)].

Upon increasing the EGP to $\sim 15 \mathrm{~mW}$, we observe that the junction traps two $1 \mu \mathrm{m}$ sized particles each time, with the front particle shifted to $z \approx 1 \mu \mathrm{m}$ [Figs. 2(e) $-2(\mathrm{~h})$ ] (Media 1). The third particle (\#3) pushes particle \#2 to substitute particle \#1.

We test a total of seven devices with the same designed dimensions on the same wafer. For the EGP $<7.5 \mathrm{~mW}$, the junction traps no particles. Within the EGP of $\sim 7.5-13 \mathrm{~mW}$, the junction traps only one $1 \mu \mathrm{m}$ sized particle. With the EGP $>13 \mathrm{~mW}$, the junction traps two $1 \mu \mathrm{m}$ sized particles. We record the longest trapping time of $\sim 30$ minutes for both one- and two-particle trapping before other waveguided particles arrive.

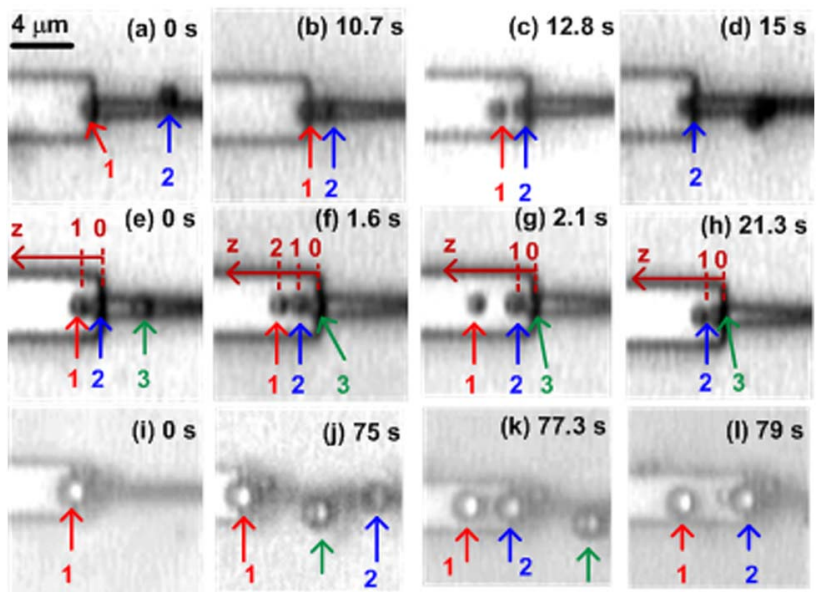

Fig. 2. (Color online) Optical micrographs of the junction with $1 \mu \mathrm{m}$ sized particles upon the EGP of (a)-(d) $\sim 12 \mathrm{~mW}$; (e)-(h) $\sim 15 \mathrm{~mW}$, (Media 1) and with (i)-(l) $2.2 \mu \mathrm{m}$ sized particles upon the EGP of $\sim 20 \mathrm{~mW}$ (green arrows label a stuck particle).

Figures 2(i)-2(l) show the optical micrographs of the $2.2 \mu \mathrm{m}$ sized particles trapping near the junction at various times upon the EGP of $\sim 20 \mathrm{~mW}$. One particle (\#1) is trapped around $z=0$ [Fig. 2(i)] before another particle (\#2) arrives [Fig. 2(j)]. Particle \#2 substitutes particle \#1 [Fig. 2(k)] and is trapped by the junction [Fig. 2(1)]. Upon increasing the EGP to $\sim 32 \mathrm{~mW}$, the number of particles trapped remains one for $2.2 \mu \mathrm{m}$ sized particles. When the EGP is reduced to $\sim 15 \mathrm{~mW}$, the junction fails to trap.

We numerically simulate the optical forces on particles in the $z$ direction as a function of particle size and position (with $0.2 \mu \mathrm{m}$ step) using finite-element-method (COMSOL RF module). At $1550 \mathrm{~nm}$ wavelength, we assume a SiN waveguide of refractive index (RI) $\sim 2.09$ with silica as the under cladding (RI of 1.44) and water as the upper cladding (RI of 1.32). We consider rigid, homogeneous, dielectric polystyrene particles (RI of 1.55). We assume a $30 \mathrm{~nm}$ gap spacing between the particle and the waveguide top-surface, following the calculated and measured Debye length in the literature [2,13]. The rest of the parameters follow the design parameters. Figure 1(b) shows the simulated mode-field amplitude distribution $30 \mathrm{~nm}$ above the $\mathrm{SiN}$ layer.

Figure 3(a) shows the simulated optical forces $\left(F_{\text {oz }}\right)$ of various sized particles (from 1 to $2.2 \mu \mathrm{m}$ in diameter) around the junction. We calculate the optical forces by integrating the Maxwell stress tensor over the particle surface $[1,14]$ and normalized to the input-waveguided power. The simulation results suggest that $F_{\mathrm{oz}}>0$ in the taper region $(z<0)$ and thus driving the particles in the taper toward the junction. In the multimode region near the junction $(z>0), F_{\mathrm{oz}}$ monotonically reduces and reaches zero around $z=0.4 \mu \mathrm{m}$ for particle size from 1 to $1.8 \mu \mathrm{m}$, suggesting particle trapping near the junction. This is consistent with our experimental observation of trapping single $1 \mu \mathrm{m}$ sized particles [Figs. 2(a)-2(d)].

However, $F_{\text {oz }}$ remains positive for $2.2 \mu$ m sized particles, indicating no particle trapping. This is inconsistent with our observation of trapping $2.2 \mu \mathrm{m}$ sized particles [Figs. 2(i)-2(1)]. In order to account for this inconsistency, we simulate $F_{\text {oz }}$ upon $2.2 \mu$ m sized particles with gap 

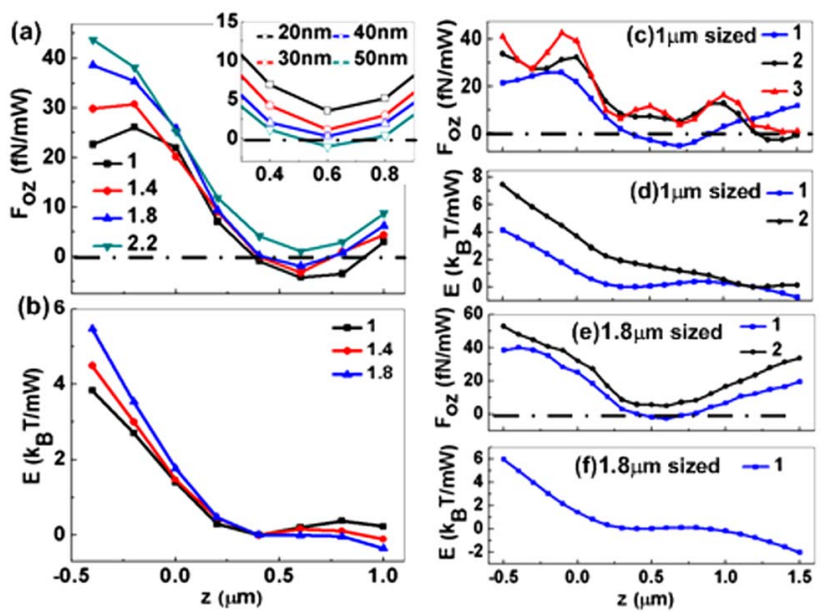

Fig. 3. (Color online) (a) Simulated $F_{\mathrm{oz}}$ on various sized particles over particle position $z$. (Inset) $F_{\mathrm{oz}} F_{\mathrm{oz}}$ on a $2.2 \mu \mathrm{m}$ sized particle with various gap spacing. (b) Calculated potential energy for 1 to $1.8 \mu \mathrm{m}$ sized particles. (c)-(f) Calculated total $F_{\mathrm{oz}}$ and potential energy for multiple particles of sizes of (c); (d) $1 \mu \mathrm{m}$ and (e), (f) $1.8 \mu \mathrm{m}$; Horizontal dotted-dashed line denotes $F_{\mathrm{oz}}=0$.

spacing of 20, 30, 40, and $50 \mathrm{~nm}$ [Fig. 3(a) inset]. For $50 \mathrm{~nm}$ gap spacing, our simulation suggests particle trapping around $z=0.5 \mu \mathrm{m}$. We also attribute the inconsistency to the water absorption at the $1550 \mathrm{~nm}$ wavelength, which introduces extra optical losses along the junction and thus results in a reduced scattering force and an additional gradient force [15]. This could potentially enable trapping larger-sized particles than we expected.

We calculate the potential energy for the trapped particles $\left(1\right.$ to $1.8 \mu \mathrm{m}$ ) near the junction by integrating $F_{\text {oz }}$ with displacement from the local equilibrium along the $z$-axis. According to the simulated optical force curve, we assume the potential energy $(E)$ at the first $F_{\text {oz }}=0$ to be zero and normalized to thermal energy $\left(k_{B} T\right)$ at room temperature $(T=300 \mathrm{~K})$ [Fig. 3(b)]. The potential energy barrier reduces as the particle size increases.

The potential energy barrier also depends on the waveguided power. From our experiments of trapping $1 \mu \mathrm{m}$ sized particles upon the EGP of $\sim 12 \mathrm{~mW}$ [Figs. 2(a)-2(d)], we estimate from the simulated potential energy a barrier height of $d E \sim 4.6 k_{B} T$ [Fig. 3(b)]. Here we assume $d E>1 k_{B} T$ as the minimum trapping criterion [9].

Given the kinetic energy of $1 \mu \mathrm{m}$ sized polystyrene particles with a velocity of $\sim 10 \mu \mathrm{m} / \mathrm{s}$ (Media 1 ) is $\sim 4-5$ orders of magnitude less than $k_{B} T$, it is unlikely that a trapped particle is knocked out by an incoming particle.

We also calculate the total optical force on multiple particles, which are in contact with each other. In the calculation, we consider the center of the front-most particle as the particle position. We obtain the total optical force by integrating the Maxwell stress tensor over the surfaces of all the particles.

Figures 3(c)-3(f) show the simulated total optical forces and the corresponding potential energy of multiple particles with sizes of 1 and $1.8 \mu \mathrm{m}$.
Figure 3(c) suggests that the junction can trap up to two $1 \mu \mathrm{m}$ sized particles with the trapping position shifts to around $z \approx 1.2 \mu \mathrm{m}$. This is consistent with our experiment of trapping two $1 \mu \mathrm{m}$ sized particles with the trapping position around $z=1 \mu \mathrm{m}$ [Figs. 2(e)-2(h)]. Based on the EGP of $\sim 15 \mathrm{~mW}$, we estimate the potential energy barrier $d E$ to be $\sim 1.9 k_{B} T$ [Fig. 3(d)], which satisfies the particle trapping criterion. For three $1 \mu \mathrm{m}$ sized particles, $F_{\text {oz }}>0$, suggesting no trapping.

Upon increasing the particle size to $1.8 \mu \mathrm{m}$, our simulation suggests that the junction can only trap one $1.8 \mu \mathrm{m}$ sized particles [Figs. 3(e)-3(f)].

In conclusion, we demonstrated optical particle trapping and successive substitution using SiN taperedwaveguide junctions. The junction trapped up to one $2.2 \mu \mathrm{m}$ sized and two $1 \mu \mathrm{m}$ sized polystyrene particles. Our experiments and numerical simulations revealed that the junction particle trapping depends on particle size and number. The simple design and broadband nature of the waveguide-junctions do not impose sophisticated designs or wavelength-tunable lasers, and thus it could serve as a simple building block for "optical tweezers circuits." However, we remark that the trapping stiffness is low comparing to resonator-based particle traps $[\underline{7}, \underline{8}]$. We expect that by increasing the guided optical power and optimizing the tapered-junction design can help enhance the trapping stiffness.

This study was substantially supported by a grant from the Research Grants Council of The Hong Kong Special Administrative Region, China, under project no. 618308. The authors acknowledge the HKUST Nanoelectronics Fabrication Facility for fabricating the optofluidic chip and Mr. Ting Lei for discussion.

\section{References}

1. S. Gaugiran, S. Getin, J. Fedeli, G. Colas, A. Fuchs, F. Chatelain, and J. Derouard, Opt. Express 13, 6956 (2005).

2. S. Lin and K. B. Crozier, Opt. Express 20, 3367 (2012).

3. H. Cai and A. W. Poon, Opt. Lett. 35, 2855 (2010).

4. D. Erickson, X. Serey, Y. F. Chen, and S. Mandal, Lab Chip 11, 995 (2011).

5. S. Lin and K. B. Crozier, Lab Chip 11, 4047 (2011).

6. H. Cai and A. W. Poon, Opt. Lett. 36, 4257 (2011).

7. Y. F. Chen, X. Serey, R. Sarkar, P. Chen, and D. Erickson, Nano Lett. 12, 1633 (2012).

8. S. Mandal, X. Serey, and D. Erickson, Nano Lett. 10, 99 (2010).

9. A. Ashkin, J. M. Dziedzic, J. E. Bjorkholm, and S. Chu, Opt. Lett. 11, 288 (1986).

10. N. Simpson, D. McGloin, K. Dholakia, L. Allen, and M. Padgett, J. Mod. Opt. 45, 1943 (1998).

11. M. MacDonald, L. Paterson, K. Volke-Sepulveda, J. Arlt, W. Sibbett, and K. Dholakia, Science 296, 1101 (2002).

12. K. Dholakia and T. Cizmar, Nat. Photon. 5, 335 (2011).

13. E. Thormann, A. Simonsen, P. Hansen, and O. Mouritsen, Langmuir 24, 7278 (2008).

14. I. Brevik, Physics Reports 52, 133 (1979).

15. X. Serey, S. Mandal, Y. F. Chen, and D. Erickson, Phys. Rev. Lett. 108, 48102 (2012). 